\title{
Energy Functions in Box Ball Systems
}

\author{
Kaori Fukuda*, Masato Okado ${ }^{\dagger}$ and Yasuhiko Yamada*
}

\begin{abstract}
The box ball system is studied in the crystal theory formulation. New conserved quantities and the phase shift of the soliton scattering are obtained by considering the energy function (or $H$-function) in the combinatorial $R$-matrix.
\end{abstract}

\section{Introduction}

The box ball system (BBS) [TS, T] is one of the important classes of soliton cellular automata, namely integrable discrete dynamical systems with solitons. Recently, an interesting relation between the BBS and the crystal theory $\mathbb{K}$, KMN has been revealed. In particular, it is proved in TNS that the scattering rule of two solitons in BBS is identical with the combinatorial $R$-matrix [NY]. Motivated by this and the work HIK, the BBS is reformulated and generalized [HKT] in terms of the crystal theory. In this formulation, the time evolution is described by the row-to-row transfer matrix obtained as a product of the combinatorial $R$-matrices.

In the affine crystal theory, the combinatorial $R$-matrix consists of the 'isomorphism' and the 'energy function' or $H$-function. In the previous works, however, the $H$-function was not taken into account. In this paper, we study the BBS based on the crystal formulation and clarify the role of the $H$-function. The main results are as follows:

- The phase shift of the soliton scattering is described by the $H$-function.

- New conserved quantities are obtained by including the $H$-function into the row-to-row transfer matrices.

In the simplest BBS, that corresponds to the $n=2$ case in our paper, it is known TTS that a Young diagram obtained by the Schensted bumping procedure $[\mathrm{F}]$ is conserved under the time evolution. Their conserved quantities

\footnotetext{
*Department of Mathematics, Faculty of Science, Kobe University, Rokko, Kobe 657-8501, Japan

$\dagger$ Department of Informatics and Mathematical Science, Graduate School of Engineering Science, Osaka University, Toyonaka, Osaka 560-8531, Japan
} 
are related to the number of solitons, and the quantities in this paper can be regarded as a generalization to theirs. We note that equivalent conserved quantities are also obtained from those of the hungry Toda molecule equation through ultra-discretization [TNS], [N].

The paper is organized as follows. In section 2, we recapitulate necessary facts from the crystal theory and reformulate the BBS. In section 3, we construct conserved quantities. The main theorem is given in section 4 , where the scattering of solitons is studied. Section 5 is devoted for discussions.

\section{Preliminaries}

In this section we recapitulate necessary facts for the $U_{q}^{\prime}\left(\widehat{\mathfrak{s l}}_{n}\right)$-crystal $B_{l}$ and then reformulate the box ball system.

\subsection{Crystal $B_{l}$}

Fix an integer $n \in \mathbf{Z}_{\geq 2}$. For an arbitrary $l \in \mathbf{Z}_{>0}$ we set

$$
B_{l}=\left\{\left(\nu_{1}, \nu_{2}, \cdots, \nu_{l}\right) \mid \nu_{j} \in\{1,2, \cdots, n\}, \nu_{1} \leq \nu_{2} \leq \cdots \leq \nu_{l}\right\}
$$

$B_{l}$ can be identified with the set of semi-standard tableaux of shape $(l)$ with letters in $\{1,2, \cdots, n\}$. For $i=0,1, \cdots, n-1$ we introduce operators $e_{i}, f_{i}: B_{l} \longrightarrow$ $B_{l} \sqcup\{0\}$ by the following rule. For $i=1, \cdots, n-1, e_{i}\left(\nu_{1}, \nu_{2}, \cdots, \nu_{l}\right)$ is obtained by replacing the leftmost $i+1$ in $\left(\nu_{1}, \nu_{2}, \cdots, \nu_{l}\right)$ with $i$ and $f_{i}\left(\nu_{1}, \nu_{2}, \cdots, \nu_{l}\right)$ by replacing the rightmost $i$ with $i+1$. If we cannot find such letter to be replaced, the result of the action should be considered as 0 . For $i=0$ the actions are given by

$$
\begin{aligned}
& e_{0}\left(\nu_{1}, \nu_{2}, \cdots, \nu_{l}\right)=\delta_{\nu_{1} 1}\left(\nu_{2}, \cdots, \nu_{l}, n\right), \\
& f_{0}\left(\nu_{1}, \nu_{2}, \cdots, \nu_{l}\right)=\delta_{\nu_{l} n}\left(1, \nu_{1}, \cdots, \nu_{l-1}\right) .
\end{aligned}
$$

Notice if $f_{i} b=b^{\prime}$ for $b, b^{\prime} \in B_{l}$, then $b=e_{i} b^{\prime} . B_{l}$ is the crystal base [K] of the $l$-th symmetric tensor representation of the quantum affine algebra $U_{q}^{\prime}\left(\widehat{\mathfrak{s l}}_{n}\right)$. For precise definitions, see [K, KMN].

Example 2.1 $n=4, l=5$

$$
\begin{aligned}
& e_{2}(11334)=(11234) \\
& f_{2}(11334)=0 \\
& f_{0}(11334)=(11133)
\end{aligned}
$$

Drawing a line from $b$ to $b^{\prime}$ with color $i$ as $b \stackrel{i}{\longrightarrow} b^{\prime}, B_{l}$ turns out a colored oriented graph called crystal graph. 
Example 2.2 (1) n: arbitrary

$B_{1}$ :

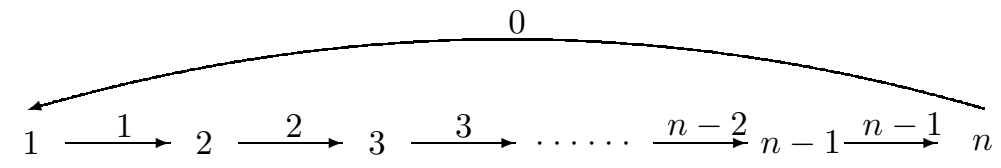

(2) $n=3$

$B_{2}$ :

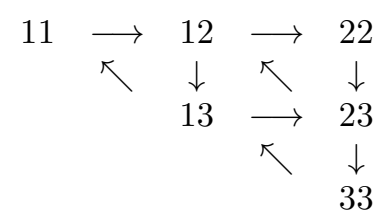

Here the colors of the arrows $\longrightarrow, \downarrow, \nwarrow$ are $1,2,0$, respectively.

\subsection{Tensor product}

For $b \in B_{l}$ we define

$$
\varepsilon_{i}(b)=\max \left\{m \geq 0 \mid e_{i}^{m} b \neq 0\right\}, \quad \varphi_{i}(b)=\max \left\{m \geq 0 \mid f_{i}^{m} b \neq 0\right\} .
$$

If $b=\left(\nu_{1}, \cdots, \nu_{l}\right) \in B_{l}, \varepsilon_{i}(b)$ (resp. $\left.\varphi_{i}(b)\right)$ is the number of $i+1$ (resp. $i+n \delta_{i 0}$ ) in $\left(\nu_{1}, \cdots, \nu_{l}\right)$. For crystals $B_{l}$ and $B_{l^{\prime}}$ the tensor product $B_{l} \otimes B_{l^{\prime}}=\left\{b \otimes b^{\prime} \mid\right.$ $\left.b \in B_{l}, b^{\prime} \in B_{l^{\prime}}\right\}$ is defined. The operators $e_{i}, f_{i}$ act on $B_{l} \otimes B_{l^{\prime}}$ as

$$
\begin{aligned}
& e_{i}\left(b \otimes b^{\prime}\right)= \begin{cases}e_{i} b \otimes b^{\prime} & \text { if } \varphi_{i}(b) \geq \varepsilon_{i}\left(b^{\prime}\right) \\
b \otimes e_{i} b^{\prime} & \text { if } \varphi_{i}(b)<\varepsilon_{i}\left(b^{\prime}\right),\end{cases} \\
& f_{i}\left(b \otimes b^{\prime}\right)= \begin{cases}f_{i} b \otimes b^{\prime} & \text { if } \varphi_{i}(b)>\varepsilon_{i}\left(b^{\prime}\right) \\
b \otimes f_{i} b^{\prime} & \text { if } \varphi_{i}(b) \leq \varepsilon_{i}\left(b^{\prime}\right) .\end{cases}
\end{aligned}
$$

Here $b \otimes 0$ and $0 \otimes b^{\prime}$ are understood to be 0 . Two crystals $B_{l} \otimes B_{l^{\prime}}, B_{l^{\prime}} \otimes B_{l}$ are known to be isomorphic, i.e. the crystal graphs are the same. We denote the image of $b \otimes b^{\prime}$ under this isomorphism by $\tilde{b}^{\prime} \otimes \tilde{b}$. Namely, we have

$$
\begin{aligned}
B_{l} \otimes B_{l^{\prime}} & \simeq B_{l^{\prime}} \otimes B_{l} \\
b \otimes b^{\prime} & \mapsto \tilde{b}^{\prime} \otimes \tilde{b} .
\end{aligned}
$$

We introduce so-called 'signature rule' to calculate the actions of $e_{i}, f_{i}$ on the tensor product. It is most effective on the multi-component tensor product. 
Consider an element $b_{1} \otimes b_{2} \otimes \cdots \otimes b_{m}$ of $B_{l_{1}} \otimes B_{l_{2}} \otimes \cdots \otimes B_{l_{m}}$. To this element we associate an $i$-signature:

$$
\eta_{i}=\underbrace{-\cdots-}_{\varepsilon_{i}\left(b_{1}\right)} \underbrace{+\cdots+}_{\varphi_{i}\left(b_{1}\right)} \underbrace{-\cdots-}_{\varepsilon_{i}\left(b_{2}\right)} \underbrace{+\cdots+}_{\varphi_{i}\left(b_{2}\right)} \cdots \underbrace{-\cdots}_{\varepsilon_{i}\left(b_{m}\right)} \underbrace{+\cdots+}_{\varphi_{i}\left(b_{m}\right)}
$$

Set $\eta_{i}^{(0)}=\eta_{i}$. We construct $\eta_{i}^{(k)}$ from $\eta_{i}^{(k-1)}$ by deleting an adjacent $(+-)$ pair in $\eta_{i}^{(k-1)}(k=1,2, \cdots)$. Then we end up with the signature of form $\bar{\eta}_{i}=$ $\underbrace{-\cdots-}_{\alpha} \underbrace{+\cdots+}_{\beta}(\alpha, \beta \geq 0)$. We call such signature reduced. Of course, there are many ways of deleting $(+-)$ pairs, but the reduced signature does not depend on them. Now the action of $e_{i}$ (resp. $f_{i}$ ) on $B_{l_{1}} \otimes B_{l_{2}} \otimes \cdots \otimes B_{l_{m}}$ is obtained by changing the rightmost - (resp. leftmost + ) of $\bar{\eta}_{i}$ to + (resp. - ). If there is no sign to be changed, the action is understood as 0 . It is easy to see that in the case of 2 components, this rule agrees with (2.1),(2.2). For details, see next example.

Example 2.3 $n=4, m=3$

Consider an element $b=(1223) \otimes(112) \otimes(24) \in B_{4} \otimes B_{3} \otimes B_{2}$. The 1signature is given as follows.

$$
\begin{aligned}
& b=(1223) \otimes(112) \otimes(24) \\
& \eta_{1}=--+\quad-++
\end{aligned}
$$

The reduced signature is

$$
\bar{\eta}_{1}=\begin{array}{ccc}
1 & 1 & 2 \\
- & - & +
\end{array}
$$

where the upper number signifies the component of the tensor product the sign belonged to. Therefore, we have

$$
\begin{aligned}
& e_{1} b=e_{1}(1223) \otimes(112) \otimes(24)=(1123) \otimes(112) \otimes(24), \\
& f_{1} b=(1223) \otimes f_{1}(112) \otimes(24)=(1223) \otimes(122) \otimes(24) .
\end{aligned}
$$

\subsection{Energy function}

Next consider a $\mathbf{Z}$-valued function $H$ on $B_{l} \otimes B_{l^{\prime}}$ satisfying the following property: For any $b \in B_{l}, b^{\prime} \in B_{l^{\prime}}$ and $i$ such that $e_{i}\left(b \otimes b^{\prime}\right) \neq 0$,

$$
H\left(e_{i}\left(b \otimes b^{\prime}\right)\right)= \begin{cases}H\left(b \otimes b^{\prime}\right)+1 & \text { if } i=0, \varphi_{0}(b) \geq \varepsilon_{0}\left(b^{\prime}\right), \varphi_{0}\left(\tilde{b}^{\prime}\right) \geq \varepsilon_{0}(\tilde{b}), \\ H\left(b \otimes b^{\prime}\right)-1 & \text { if } i=0, \varphi_{0}(b)<\varepsilon_{0}\left(b^{\prime}\right), \varphi_{0}\left(\tilde{b}^{\prime}\right)<\varepsilon_{0}(\tilde{b}), \\ H\left(b \otimes b^{\prime}\right) & \text { otherwise }\end{cases}
$$

$H$ is known to exist and unique up to additive constant. The existences of the isomorphism (2.3) and energy function $H$ are guaranteed by the existence of the $R$-matrix. See [KMN]. 
Explicit rules to calculate the isomorphism and energy function are obtained in $\left[\mathrm{NY}\right.$. Here we summarize the procedure to obtain them. We assume $l \geq l^{\prime}$. Let $b \otimes b^{\prime}$ be an element in $B_{l} \otimes B_{l^{\prime}}$ such as $b=\left(\nu_{1}, \ldots, \nu_{l}\right)$ and $b^{\prime}=\left(\nu_{1}^{\prime}, \ldots, \nu_{l^{\prime}}^{\prime}\right)$. Let $x_{i}$ (resp. $y_{i}$ ) be the number of $i$ in $b$ (resp. $b^{\prime}$ ). We represent $b \otimes b^{\prime}$ by the two column diagram. Each column has $n$ rows, enumerated as 1 to $n$ from the top to the bottom. We put $x_{i}$ (resp. $y_{i}$ ) letters $i$ in the $i$-th row of the left (resp. right) column. The rule to obtain the energy function $H$ and the isomorphism is as follows.

(1) Pick any letter, say $i$, in the right column and connect it with a letter $j$ in the left column by a line. The partner $j$ is chosen so that $j=$ $\max \left\{\nu_{k} \mid \nu_{k}<i\right\}$. If there is no such $j$, we return to the bottom and the partner $j$ is chosen so that $j=\max \left\{\nu_{k}\right\}$. In the latter case, we call such a pair or line "winding".

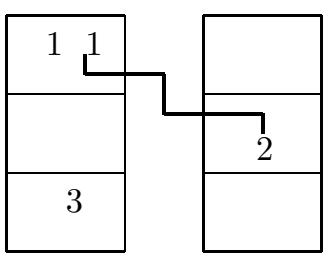

unwinding

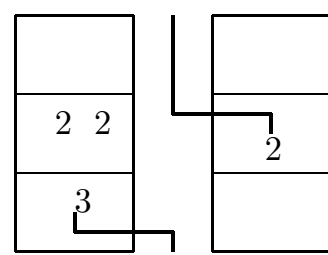

winding

(2) Repeat the procedure (1) for the remaining unconnected letters $(l-1)$ times.

(3) The isomorphism is obtained by sliding the remaining $(k-l)$ unpaired letters in the left column to the right.

(4) The value of the energy function is $(-1)$ times the number of the "unwinding" pairs.

Note that we normalized $H$ so that we have $H\left(\left(n^{l}\right) \otimes\left(n^{l^{\prime}}\right)\right)=0$. Here and later, $\left(n^{l}\right)$ means $(n, n, \cdots, n) \in B_{l}$. When $l^{\prime}=1$, the rule simplifies to the following:

If there exists $k$ such that $\nu_{k}<\nu^{\prime}$, then

$$
\begin{aligned}
& \left(\nu_{1}, \cdots, \nu_{l}\right) \otimes\left(\nu^{\prime}\right) \mapsto\left(\nu_{j}\right) \otimes\left(\nu_{1}, \cdots, \nu^{\prime}, \cdots, \nu_{l}\right), \\
& H\left(\left(\nu_{1}, \cdots, \nu_{l}\right) \otimes\left(\nu^{\prime}\right)\right)=-1,
\end{aligned}
$$

where $j=\max \left\{k \mid \nu_{k}<\nu^{\prime}\right\}$. Otherwise,

$$
\begin{aligned}
& \left(\nu_{1}, \cdots, \nu_{l}\right) \otimes\left(\nu^{\prime}\right) \mapsto\left(\nu_{l}\right) \otimes\left(\nu^{\prime}, \nu_{1}, \cdots, \nu_{l-1}\right), \\
& H\left(\left(\nu_{1}, \cdots, \nu_{l}\right) \otimes\left(\nu^{\prime}\right)\right)=0 .
\end{aligned}
$$


Example 2.4 $l=4, l^{\prime}=2$

$$
\begin{aligned}
& (1123) \otimes(23) \simeq(12) \otimes(1233) \quad H=-2 \\
& (1123) \otimes(12) \simeq(13) \otimes(1122) \quad H=-1 \\
& (2344) \otimes(12) \simeq(44) \otimes(1223) \quad H=0
\end{aligned}
$$

\subsection{Yang-Baxter equation}

Let us define the affinization $A f f\left(B_{l}\right)$ of the crystal $B_{l}$. We introduce an indeterminate $z$ (the spectral parameter) and set

$$
\operatorname{Aff}\left(B_{l}\right)=\left\{z^{d} b \mid d \in \mathbf{Z}, b \in B_{l}\right\} .
$$

Thus $\operatorname{Aff}\left(B_{l}\right)$ is an infinite set. $z^{0} b \in A f f\left(B_{l}\right)$ will often be written as $b$.

Definition 2.5 A combinatorial $R$-matrix for a crystal $B_{l} \otimes B_{l^{\prime}}$ is a map $R$ : $\operatorname{Aff}\left(B_{l}\right) \otimes \operatorname{Aff}\left(B_{l^{\prime}}\right) \longrightarrow \operatorname{Aff}\left(B_{l^{\prime}}\right) \otimes \operatorname{Aff}\left(B_{l}\right)$ given by

$$
R\left(z^{d} b \otimes z^{d^{\prime}} b^{\prime}\right)=z^{d^{\prime}+H\left(b \otimes b^{\prime}\right)} \tilde{b}^{\prime} \otimes z^{d-H\left(b \otimes b^{\prime}\right)} \tilde{b} .
$$

The following result is a direct consequence of the ordinary (i.e. not combinatorial) Yang-Baxter equation.

Proposition 2.6 (Yang-Baxter equation) The following equation holds on $\operatorname{Aff}\left(B_{l}\right) \otimes \operatorname{Aff}\left(B_{l^{\prime}}\right) \otimes \operatorname{Aff}\left(B_{l^{\prime \prime}}\right)$.

$$
(R \otimes 1)(1 \otimes R)(R \otimes 1)=(1 \otimes R)(R \otimes 1)(1 \otimes R)
$$

\subsection{Box ball system}

Here and in what follows, we set $B=B_{1}$ and consider the crystal $B^{\otimes L}$ for sufficiently large $L$. The elements of $B^{\otimes L}$ we have in mind are of the following form.

$\cdots \otimes(n) \otimes(n) \otimes \cdots \otimes \otimes(n) \otimes\left(\nu_{1}\right) \otimes\left(\nu_{2}\right) \otimes \cdots \otimes\left(\nu_{k}\right) \otimes(n) \otimes(n) \otimes \cdots \otimes(n) \otimes \cdots$,

where $\nu_{1}, \nu_{2}, \cdots, \nu_{k} \in\{1,2, \cdots, n\}$. Namely, relatively few elements are non $(n)$, and almost all are $(n)$. In the propositions below, we embed, if necessary, $B^{\otimes L}$ into $B^{\otimes L^{\prime}}\left(L<L^{\prime}\right)$ by

$$
\begin{aligned}
B^{\otimes L} & \hookrightarrow B^{\otimes L^{\prime}} \\
b_{1} \otimes \cdots \otimes b_{L} & \mapsto b_{1} \otimes \cdots \otimes b_{L} \otimes \underbrace{(n) \otimes \cdots \otimes(n)}_{L^{\prime}-L} .
\end{aligned}
$$


Lemma 2.7 By iterating the isomorphism $B_{l} \otimes B \simeq B \otimes B_{l}$, we consider a map

$$
\begin{aligned}
B_{l} \otimes B \otimes \cdots \otimes B & \stackrel{\sim}{\longrightarrow} B \otimes \cdots \otimes B \otimes B_{l} \\
\left(n^{l}\right) \otimes b_{1} \otimes \cdots \otimes b_{L} & \mapsto \tilde{b}_{1} \otimes \cdots \otimes \tilde{b}_{L} \otimes \tilde{b} .
\end{aligned}
$$

Then there exists an integer $L_{0}$ such that $\tilde{b}=\left(n^{l}\right)$ for $L \geq L_{0}$.

Taking sufficiently large $L$ such that the above lemma holds, we define a map $T_{l}: B^{\otimes L} \longrightarrow B^{\otimes L}$ by $b_{1} \otimes \cdots \otimes b_{L} \mapsto \tilde{b}_{1} \otimes \cdots \otimes \tilde{b}_{L}$.

Lemma 2.8 For a fixed element of $B^{\otimes L}$, there exists an integer $l_{0}$ such that $T_{l}=T_{l_{0}}$ for any $l \geq l_{0}$.

Both lemmas are obvious from (2.4), (2.5).

Following [HKT], we reformulate the box ball system. We consider such an element of $B^{\otimes L}$, often called a 'state', as described in the beginning of this subsection. Lemma 2.7 and 2.8 enables us to define an operator $T=\lim _{l \rightarrow \infty} T_{l}$ on the space of states. Application of $T$ gives rise to a transition of state. Thus it can be regarded as describing a certain dynamical system, in which $T$ plays the role of 'time evolution'. From the same reason, $T_{l}$ may also be viewed as a time evolution. (In this paper, time evolution means the one by $T$ unless otherwise stated.)

Under the condition assumed in Lemma 2.7, the sequence $b_{1} \otimes \cdots \otimes b_{L}$ is determined uniquely from $\tilde{b}_{1} \otimes \cdots \otimes \tilde{b}_{L}$ and $b=\left(n^{l}\right)$, since the isomorphisms $B_{l} \otimes B \simeq B \otimes B_{l}$ are bijective. Hence, the time evolutions $T_{l}(1 \geq l)$ are invertible. The inverse map of the isomorphism (2.4), (2.5) is given explicitly by the following rule.

If there exists $k$ such that $\nu_{k}>\nu^{\prime}$, then

$$
\left(\nu^{\prime}\right) \otimes\left(\nu_{1}, \cdots, \nu_{l}\right) \mapsto\left(\nu_{1}, \cdots, \nu^{\prime}, \cdots, \nu_{l}\right) \otimes\left(\nu_{j}\right),
$$

where $j=\min \left\{k \mid \nu_{k}>\nu^{\prime}\right\}$. Otherwise,

$$
\left(\nu^{\prime}\right) \otimes\left(\nu_{1}, \cdots, \nu_{l}\right) \mapsto\left(\nu_{2}, \cdots, \nu_{l}, \nu^{\prime}\right) \otimes\left(\nu_{1}\right) .
$$

As we see in the next section, these time evolutions $T_{l}(l \geq 1)$ form a commuting family of operators and play an important role in our paper.

\section{Conservation laws}

In this section we construct conserved quantities under the time evolutions. 


\subsection{Definition of $E_{l}$}

Fix sufficiently large $L$ and consider a composition of the combinatorial $R$ matrices

$$
\mathcal{R}_{l}=R_{L L+1} \cdots R_{23} R_{12}: \operatorname{Aff}\left(B_{l}\right) \otimes \operatorname{Aff}(B)^{\otimes L} \longrightarrow \operatorname{Aff}(B)^{\otimes L} \otimes \operatorname{Aff}\left(B_{l}\right) .
$$

Here $R_{i+1}$ signifies that the $R$-matrix acts on the $i$-th and $(i+1)$-th components of the tensor product. Applying $\mathcal{R}_{l}$ to an element $\left(n^{l}\right) \otimes p\left(p=b_{1} \otimes \cdots \otimes b_{L}\right)$, we have

$$
\begin{aligned}
\mathcal{R}_{l}\left(\left(n^{l}\right) \otimes p\right) & =z^{H_{1}} \tilde{b}_{1} \otimes z^{H_{2}} \tilde{b}_{2} \otimes \cdots \otimes z^{H_{L}} \tilde{b}_{L} \otimes z^{E_{l}(p)}\left(n^{l}\right), \\
E_{l}(p) & =-\sum_{j=1}^{L} H_{j}, \quad H_{j}=H\left(b^{(j-1)} \otimes b_{j}\right),
\end{aligned}
$$

where $b^{(j)}(0 \leq j<L)$ is defined by

$$
\begin{gathered}
B_{l} \otimes \underbrace{B \otimes \cdots \otimes B}_{j} \simeq \underbrace{B \otimes \cdots \otimes B}_{j} \otimes B_{l} \\
\left(n^{l}\right) \otimes b_{1} \otimes \cdots \otimes b_{j} \mapsto \tilde{b}_{1} \otimes \cdots \otimes \tilde{b}_{j} \otimes b^{(j)} .
\end{gathered}
$$

\subsection{Conservation of $E_{l}$}

Proposition 3.1 As maps from Aff $\left(B_{l}\right) \otimes A f f\left(B_{l^{\prime}}\right) \otimes A f f(B)^{\otimes L}$ to Aff $(B)^{\otimes L} \otimes$ $\operatorname{Aff}\left(B_{l^{\prime}}\right) \otimes \operatorname{Aff}\left(B_{l}\right)$, the following are identical.

$$
\mathcal{R}_{l^{\prime}} \mathcal{R}_{l} R_{12}=R_{L+1 L+2} \mathcal{R}_{l} \mathcal{R}_{l^{\prime}}
$$

Here $\mathcal{R}_{l}$ (resp. $\left.\mathcal{R}_{l^{\prime}}\right)$ acts on $\operatorname{Aff}\left(B_{l}\right) \otimes \operatorname{Aff}(B)^{\otimes L}$ (resp. Aff $\left.\left(B_{l^{\prime}}\right) \otimes \operatorname{Aff}(B)^{\otimes L}\right)$, and acts on the other component as identity.

Proof. Use the Yang-Baxter equation repeatedly.

Theorem 3.2 For an element $p \in B^{\otimes L}$, we have

(1) $T_{l} T_{l^{\prime}}(p)=T_{l^{\prime}} T_{l}(p)$.

(2) $E_{l}\left(T_{l^{\prime}}(p)\right)=E_{l}(p)$. In particular, $E_{l}(T(p))=E_{l}(p)$.

Proof. Consider an element $\left(n^{l}\right) \otimes\left(n^{l^{\prime}}\right) \otimes p\left(p=b_{1} \otimes \cdots \otimes b_{L}\right)$ of $\operatorname{Aff}\left(B_{l}\right) \otimes$ $\operatorname{Aff}\left(B_{l^{\prime}}\right) \otimes \operatorname{Aff}(B)^{\otimes L}$. Apply both sides of (3.1) to it. Then one gets

$$
\begin{aligned}
& L H S=z^{H_{1}+\tilde{H}_{1}} \tilde{\tilde{b}}_{1} \otimes \cdots \otimes z^{H_{L}+\tilde{H}_{L}} \tilde{\tilde{b}}_{L} \otimes z^{E_{l^{\prime}}(\tilde{p})}\left(n^{l^{\prime}}\right) \otimes z^{E_{l}(p)}\left(n^{l}\right), \\
& R H S=z^{H_{1}^{\prime}+\hat{H}_{1}} \hat{\hat{b}}_{1} \otimes \cdots \otimes z^{H_{L}^{\prime}+\hat{H}_{L}} \hat{\hat{b}}_{L} \otimes z^{E_{l^{\prime}}(p)}\left(n^{l^{\prime}}\right) \otimes z^{E_{l}(\hat{p})}\left(n^{l}\right) .
\end{aligned}
$$

Here $\tilde{\tilde{b}}_{1} \otimes \cdots \otimes \tilde{\tilde{b}}_{L}=T_{l^{\prime}} T_{l}(p), \hat{\hat{b}}_{1} \otimes \cdots \otimes \hat{\hat{b}}_{L}=T_{l} T_{l^{\prime}}(p)$ and $\tilde{p}=T_{l}(p), \hat{p}=T_{l^{\prime}}(p)$. $H_{j}, \tilde{H}_{j}, H_{j}^{\prime}, \hat{H}_{j}(1 \leq j \leq L)$ are values of the energy functions, which are not necessary here. (3.1) obviously ensures (1) and (2). 


\section{Solitons}

\subsection{Soliton}

A state of the following form is called an $m$-soliton state of length $l_{1}, l_{2}, \ldots, l_{m}$,

$$
\ldots\left[l_{1}\right] \ldots \ldots . .\left[l_{2}\right] \ldots \ldots \ldots \ldots\left[l_{m}\right] \ldots \ldots .
$$

Here ..[l].. denotes a local configuration such as

$$
\cdots \otimes n \otimes n \otimes \underbrace{\nu_{1} \otimes \nu_{2} \otimes \cdots \otimes \nu_{l}}_{l} \otimes n \otimes n \otimes \cdots, \quad\left(n>\nu_{1} \geq \nu_{2} \geq \cdots \geq \nu_{l} \geq 1\right),
$$

sandwiched by sufficiently many $n$ 's. (In this section, the parentheses in the state are omitted.)

Lemma 4.1 Let $p$ be a 1-soliton state of length $l$, then

(1) The $k$-th conserved quantity of $p$ is given by $E_{k}(p)=\min (k, l)$.

(2) The state $T_{k}(p)$ is obtained by the rightward shift by $E_{k}(p)$ lattice steps.

Proof. (1) Recall that the conserved quantity $E_{k}$ is a sum of local $H$-functions $-H_{j}=-H\left(b^{(j-1)} \otimes b_{j}\right) \in\{0,1\}$. By the rule $(2.2)$ and $(2.3)$, one has $-H_{j}=1$ if and only if $j=J+1, J+2, \ldots, J+\min (k, l)$, where $J$ is the position of the last component $\nu_{l}$ in the tensor product $B^{L}$. Hence $E_{k}=\min (k, l)$. Similarly, the statement (2) follows from the rule (2.2) and (2.3).

Example 4.2 The time evolution円 of 1 soliton of length 3. Here and in what follows . means $n(n>3)$.

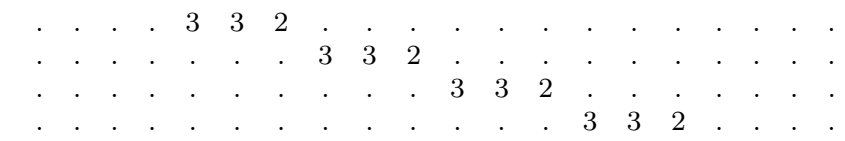

Definition 4.3 For any state $p$, the numbers $N_{l}=N_{l}(p)(l=1,2, \ldots)$ are defined by

$$
\begin{aligned}
& E_{l}=\sum_{k \geq 1} \min (k, l) N_{k}, \quad E_{0}=0, \\
& N_{l}=-E_{l-1}+2 E_{l}-E_{l+1} .
\end{aligned}
$$

By Lemma 4.1, we have

Proposition 4.4 For m-soliton state (4.1), $N_{l}$ is the number of solitons of length $l$,

$$
N_{l}=\sharp\left\{j \mid l_{j}=l\right\} .
$$

This proposition implies the stability of solitons, since the numbers $E_{l}(p)$, and hence $N_{l}(p)$, are conserved.

\footnotetext{
${ }^{1}$ The convention of the box ball game here is different from [TS, T] in that, bigger numbers are transferred before smaller ones.
} 


\section{$4.2 \quad$ Scattering of solitons}

The following is an example of the time evolution (for $t=0,1, \ldots, 6$ ) of a state which shows the scattering of three solitons of length 3,2 and 1 .

\section{Example 4.5}

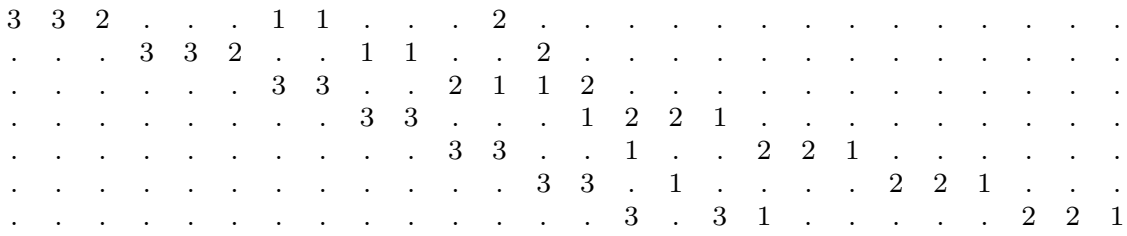

We introduce a labeling of solitons of length $l$ using $A f f\left(B_{l}\right)$ for the lower rank algebra $U_{q}^{\prime}\left(\widehat{\mathfrak{s l}}_{n-1}\right)$. (See also HKT for such an identification.) Suppose there is a soliton of length $l \cdots \otimes \nu_{1} \otimes \nu_{2} \otimes \cdots \otimes \nu_{l} \otimes \cdots\left(n>\nu_{1} \geq \nu_{2} \geq \cdots \geq \nu_{l} \geq 1\right)$ at time $t$. Say it is at position $\gamma(t)$, if $\left(\nu_{1}\right)$ is in the $\gamma(t)$-th tensor component of $B^{\otimes L}$. From Lemma 4.1 (2), the position $\gamma(t)$ under the time evolution $T_{k}$ is given by $\gamma(t)=\min (k, l) t+\gamma(\min (k, l)$ is the velocity and $\gamma$ is the phase $)$ unless it interacts with other solitons. To such a soliton we associate an element $z^{-\gamma}\left(\nu_{l}, \nu_{l-1}, \cdots, \nu_{1}\right) \in \operatorname{Aff}\left(B_{l}\right)$ for $U_{q}^{\prime}\left(\widehat{\mathfrak{s l}}_{n-1}\right)$.

Now consider a state of $m$ solitons illustrated as below.

$$
\ldots\left[l_{1}\right] \ldots .\left[l_{2}\right] \ldots \ldots \ldots \ldots \ldots\left[l_{m}\right] \ldots \ldots \ldots \ldots \ldots
$$

We assume solitons are separated enough from each other and $l_{1}>l_{2}>\cdots>l_{m}$. Since longer solitons move faster, we can expect that the state turns out to be

$$
\text { ..... }\left[l_{m}\right] \ldots \ldots \ldots . .\left[l_{2}\right] \ldots . .\left[l_{1}\right] \ldots \ldots \ldots \ldots \ldots
$$

after sufficiently many time evolutions. (The proof of this fact is found in that of the main theorem.) We represent such a scattering process as

$$
\begin{array}{r}
z^{c_{1}} b_{1} \otimes z^{c_{2}} b_{2} \otimes \cdots \otimes z^{c_{m}} b_{m} \\
\mapsto z^{c_{m}^{\prime}} b_{m}^{\prime} \otimes \cdots \otimes z^{c_{2}^{\prime}} b_{2}^{\prime} \otimes z^{c_{1}^{\prime}} b_{1}^{\prime}
\end{array}
$$

Here $z^{c_{j}} b_{j}, z^{c_{j}^{\prime}} b_{j}^{\prime}$ are elements of $A f f\left(B_{l_{j}}\right)$ under the identification in the previous paragraph. With these notations, the scattering process in Example 1.5 is described as

$$
z^{0}(2,3,3) \otimes z^{-6}(1,1) \otimes z^{-11}(2) \mapsto z^{-8}(3) \otimes z^{-4}(1,3) \otimes z^{-5}(1,2,2) .
$$

Let us recall some useful fact derived from representation theory. Note that $U_{q}^{\prime}\left(\widehat{\mathfrak{s l}}_{n}\right)$ contains $U_{q}\left(\mathfrak{s l}_{n-1}\right)$ as subalgebra. This fact can be translated into the language of crystals and guarantees that

$T_{k}$ commutes with $e_{i}, f_{i}(i=1,2, \cdots, n-2)$ on $\operatorname{Aff}\left(B_{l_{1}}\right) \otimes \cdots \otimes \operatorname{Aff}\left(B_{l_{m}}\right)$. 
Here the actions of $e_{i}, f_{i}(i=1,2, \cdots, n-2)$ on the multi-component tensor product can be calculated using signature rule explained in section 2.2. By the actions, the power of $z$ in an element of $\operatorname{Aff}\left(B_{l_{j}}\right)$ is unaffected. We call this property $U_{q}\left(\mathfrak{s l}_{n-1}\right)$-invariance. This property is also used to prove our theorem. For instance, if we admit (4.2), we have

$$
\begin{aligned}
& e_{2}\left(z^{0}(2,3,3) \otimes z^{-6}(1,1) \otimes z^{-11}(2)\right)=z^{0}(2,2,3) \otimes z^{-6}(1,1) \otimes z^{-11}(2) \\
\mapsto & e_{2}\left(z^{-8}(3) \otimes z^{-4}(1,3) \otimes z^{-5}(1,2,2)\right)=z^{-8}(3) \otimes z^{-4}(1,2) \otimes z^{-5}(1,2,2) .
\end{aligned}
$$

Let us explain the $U_{q}\left(\mathfrak{s l}_{n-1}\right)$-invariance in terms of tableau combinatorics. Consider a state, read letters from right to left and remove all $n$ 's. Suppose the resulting sequence of letters is $w=i_{1} \cdots i_{s}\left(1 \leq i_{k} \leq n-1\right)$. From such a sequence (or word) one can define a semi-standard tableau $\left(\cdots\left(\left(i_{1} \leftarrow i_{2}\right) \leftarrow\right.\right.$ $\left.\left.i_{3}\right) \cdots \leftarrow i_{s}\right)$ by the 'row bumping' algorithm $[\mathbb{E}]$. The $U_{q}\left(\mathfrak{s l}_{n-1}\right)$-invariance assures that the resulting tableau is invariant under the time evolution. In the example (4.2), the words before and after the scattering are given by $w=211233$ and $w^{\prime}=122133$, and the corresponding tableaux are the same:

$$
\begin{array}{lllll}
1 & 1 & 2 & 3 & 3 \\
2 & & & &
\end{array}
$$

Of course, this invariance is valid in the intermediate stage of scattering.

The main results of this paper are the following.

Theorem 4.6 (1) The two body scattering of solitons of length $l_{1}$ and length $l_{2}\left(l_{1}>l_{2}\right)$ under the time evolution $T_{r}\left(r>l_{2}\right)$ is described by the combinatorial R-matrix:

$$
\begin{aligned}
\operatorname{Aff}\left(B_{l_{1}}\right) \otimes \operatorname{Aff}\left(B_{l_{2}}\right) & \simeq \operatorname{Aff}\left(B_{l_{2}}\right) \otimes \operatorname{Aff}\left(B_{l_{1}}\right) \\
z^{c_{1}} b_{1} \otimes z^{c_{2}} b_{2} & \mapsto z^{c_{2}+\delta} \tilde{b}_{2} \otimes z^{c_{1}-\delta} \tilde{b}_{1},
\end{aligned}
$$

with $\delta=2 l_{2}+H\left(b_{1} \otimes b_{2}\right)$.

(2) The scattering of solitons is factorized into two body scatterings.

Some remarks may be in order.

1) The combinatorial $R$-matrix in Theorem 4.6 has an extra term $2 l_{2}$ in the power of $z$. However, the Yang-Baxter equation (Proposition 2.6) holds as it is. 2) Although we do not consider the cases where there exist solitons with same length, some part of the results can be generalized to such situations. Let us consider a state

$$
. . \underbrace{[k] \ldots[k]}_{N_{k}} \cdots \cdots \cdot . . \underbrace{[2] \ldots[[2]}_{N_{2}} \cdots \cdots \cdot \underbrace{[1] \ldots[1]}_{N_{1}} \ldots \ldots,
$$


consisting of $N_{j}$ solitons of length $j(1 \leq j \leq k)$. By the same argument given in the following proof, we can show that the scattering of these solitons is factorized into scattering of two bunches $(1 \leq j<i \leq k)$

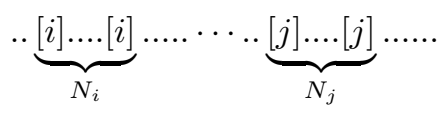

We conjecture this scattering is described by the product of $N_{i} N_{j}$ combinatorial $R$ matrices $\operatorname{Aff}\left(B_{i}\right) \otimes \operatorname{Aff}\left(B_{j}\right) \simeq \operatorname{Aff}\left(B_{j}\right) \otimes \operatorname{Aff}\left(B_{i}\right)$. This conjecture is trivially true if each solitons are separated enough.

Example 4.7 Using this theorem, the scattering process (4.2) is calculated as

$$
\begin{aligned}
& z^{0}(2,3,3) \otimes z^{-6}(1,1) \otimes z^{-11}(2)=\left\{z^{0}(2,3,3) \otimes z^{-6}(1,1)\right\} \otimes z^{-11}(2) \\
\mapsto & \left\{z^{-6+4}(3,3) \otimes z^{0-4}(1,1,2)\right\} \otimes z^{-11}(2)=z^{-2}(3,3) \otimes\left\{z^{-4}(1,1,2) \otimes z^{-11}(2)\right\} \\
\mapsto & z^{-2}(3,3) \otimes\left\{z^{-11+1}(1) \otimes z^{-4-1}(1,2,2)\right\}=\left\{z^{-2}(3,3) \otimes z^{-10}(1)\right\} \otimes z^{-5}(1,2,2) \\
\mapsto & \left\{z^{-10+2}(3) \otimes z^{-2-2}(1,3)\right\} \otimes z^{-5}(1,2,2)=z^{-8}(3) \otimes z^{-4}(1,3) \otimes z^{-5}(1,2,2) .
\end{aligned}
$$

The result is independent of the order of the factorization due to the Yang-Baxter equation.

Proof of Theorem 4.9. (1) Two soliton scattering rule: Due to the $U_{q}\left(\mathfrak{s l}_{n-1}\right)$ invariance, it is enough to check the rule for the highest weight elements $\left(1^{i}\right) \otimes$ $\left(1^{l}, 2^{k}\right) \in B_{i} \otimes B_{k+l}(i>l+k)$, i.e. elements such that $e_{j}\left(b_{1} \otimes b_{2}\right)=0$ for any $j=1, \cdots, n-2$. The corresponding state is given by

$$
\cdots \otimes \underbrace{1 \otimes \cdots \otimes 1}_{i} \otimes \underbrace{n \otimes \cdots \otimes n}_{j} \otimes \underbrace{2 \otimes \cdots \otimes 2}_{k} \otimes \underbrace{1 \otimes \cdots \otimes 1}_{l} \otimes \cdots .
$$

Now consider the time evolution by $T_{k+l+1}$. If $j>l,\left(1^{i}\right)$ moves with velocity $k+l+1$ and $\left(1^{l}, 2^{k}\right)$ with $k+l$. At some time, we arrive at the state

$$
\cdots \otimes \underbrace{1 \otimes \cdots \otimes 1}_{i} \otimes \underbrace{n \otimes \cdots \otimes n}_{l} \otimes \underbrace{2 \otimes \cdots \otimes 2}_{k} \otimes \underbrace{1 \otimes \cdots \otimes 1}_{l} \otimes \cdots .
$$

By the rule (2.4)(2.5), it is easy to see that after $t$ time units $(t \leq i-k-l)$ from this moment, the state becomes

$$
\cdots \otimes \underbrace{n \otimes \cdots \otimes n}_{(k+l+1) t} \otimes \underbrace{1 \otimes \cdots \otimes 1}_{i-t} \otimes \underbrace{n \otimes \cdots \otimes n}_{l} \otimes \underbrace{2 \otimes \cdots \otimes 2}_{k} \otimes \underbrace{1 \otimes \cdots \otimes 1}_{l+t} \otimes \cdots .
$$

After that $(t>i-k-l)$, the solitons never interact again.

We have investigated the time evolution by $T_{k+l+1}$. However, we have the equality

$$
T_{r}^{b} T_{k+l+1}^{a}=T_{k+l+1}^{a} T_{r}^{b} \quad(r>k+l) .
$$


If $a$ and $b$ are sufficiently large, we can reduce the observation of the scattering by $T_{r}$ in the right hand side to that by $T_{k+l+1}$ in the left hand side, which we have just finished. It is immediate to check that $\left(1^{i}\right) \otimes\left(1^{l}, 2^{k}\right) \mapsto\left(1^{l+k}\right) \otimes\left(1^{i-k}, 2^{k}\right)$ under the isomorphism $B_{i} \otimes B_{l+k} \simeq B_{l+k} \otimes B_{i}$. Let $\delta_{1}$ (resp. $\delta_{2}$ ) be the phase shift of the soliton of length $i$ (resp. soliton of length $(l+k)$ ). They are given by

$$
\begin{aligned}
& \delta_{1}=(k+l+1)(i-k-l)+(k+l)+l-(k+l+1)(i-k-l)=2 l+k, \\
& \delta_{2}=(k+l+1)(i-k-l)-i-l-(k+l)(i-k-l)=-(2 l+k) .
\end{aligned}
$$

Since $H\left(\left(1^{i}\right) \otimes\left(1^{l}, 2^{k}\right)\right)=-k$, we have the desired result.

(2) Factorization property: We illustrate the proof by a scattering of three solitons of length 3,2 and 1. Other cases are similar. Now the initial state is

$$
p=\ldots[3] . .[2] \ldots \ldots \ldots[1] \ldots \ldots \ldots \ldots \ldots
$$

We use a similar technique to (1). By applying the operator $T_{2}^{a}(a>>0)$ we have scatterings of [2] $\otimes[1]$ and $[3] \otimes[1]$.

$$
T_{2}^{a}(p)=\ldots \ldots \ldots[1] \ldots \ldots \ldots[3] . .[2] \ldots \ldots \ldots \ldots
$$

Then by $T_{3}^{b}(b>>0)$ we have the third scattering of $[3] \otimes[2]$.

$$
T_{3}^{b} T_{2}^{a}(p)=\ldots \ldots \ldots[1] \ldots \ldots \ldots[2] \ldots \ldots[3] \ldots \ldots \ldots \ldots
$$

After this the time evolution $T^{c}$ acts trivially. On the other hand, these evolution can be rewritten as

$$
T^{c} T_{3}^{b} T_{2}^{a}=T_{3}^{b} T_{2}^{a} T^{c}
$$

In the right hand side, we first observe three soliton scattering caused by $T^{c}$ $(c>>0)$ and then $T_{3}^{b} T_{2}^{a}$ act trivially. Hence, the three soliton scattering in the right hand side is factorized into $3 \times$ (two soliton scattering) in the left hand side.

\section{Discussion}

In this paper we have studied the box ball system in the crystal base formulation and clarified the role of $H$-functions. The $H$-function plays two important roles in the system: (1) to construct the conserved quantities and (2) to describe the phase shift of the scattering. Similar results are expected for the generalized models defined in HKT.

As far as we know, there is no direct proof of the equivalence between the original box ball systems and their crystal theoretic reformulation. This problem is under investigation.

Another important feature of the box ball systems is their relation to nonlinear integrable equations through the 'ultra discretization' [MSTTT, TTMS, 
TNS. It is an interesting problem to understand the direct relation between such nonlinear integrable equations and crystal theory.

Acknowledgements. The authors would like to thank G. Hatayama, A. Kuniba, M. Noumi, T. Takagi, T. Takebe and T. Tokihiro for valuable discussions. They also thank G. Hatayama, A. Kuniba and T. Takagi for sending HKT prior to publication.

\section{References}

[F] W. Fulton, Young tableaux, London Mathematical Society Student Texts 35, Cambridge University Press (1997).

[HKT] G. Hatayama, A. Kuniba and T. Takagi, Soliton cellular automata associated with finite crystals, preprint (1999).

[HHIKTT] G. Hatayama, K. Hikami, R. Inoue, A. Kuniba, T. Takagi and T. Tokihiro, in preparation.

[HIK] K. Hikami, R. Inoue and Y. Komori, Crystallization of the Bogoyavlensky lattice, J. Phys. Soc. Jpn. 68 (1999) 2234-2240.

[KMN] S-J. Kang, M. Kashiwara, K. C. Misra, T. Miwa, T. Nakashima and A. Nakayashiki, Affine crystals and vertex models, Int. J. Mod. Phys. A 7 (suppl. 1A), 449-484 (1992).

[K] M. Kashiwara, On crystal bases of the q-analogue of universal enveloping algebras, Duke Math. J. 63, 465-516 (1991).

[MSTTT] J. Matsukidaira, J. Satsuma, D. Takahashi, T. Tokihiro and M. Torii, Toda-type cellular automaton and its $N$-soliton solution, Phys. Lett. A 225 (1997) 287-295.

[N] A. Nagai, Talk presented at the meeting of Japan Math. Soc. (Hiroshima, 1999).

[NY] A. Nakayashiki and Y. Yamada, Kostka polynomials and energy functions in solvable lattice models, Selecta Mathematica, New Ser. 3, 547-599 (1997).

[TS] D. Takahashi and J. Satsuma, A soliton cellular automaton, J. Phys. Soc. Jpn. 59 (1990) 3514-3519.

[T] D. Takahashi, On some soliton systems defined by using boxes and balls, Proceedings of the International Symposium on Nonlinear Theory and Its Applications (NOLTA '93), (1993) 555-558. 
[TTS] M. Torii, D. Takahashi and J. Satsuma, Combinatorial representation of invariants of a soliton cellular automaton, Phys. D 92 (1996) 209-220.

[TTMS] T. Tokihiro, D. Takahashi, J. Matsukidaira and J. Satsuma, From soliton equations to integrable cellular automata through a limiting procedure, Phys. Rev. Lett. 76 (1996) 3247-3250.

[TNS] T. Tokihiro, A. Nagai and J. Satsuma, Proof of solitonical nature of box and ball systems by means of inverse ultra-discretization, preprint (1999). 